\title{
ASCILITE 2021
}

Back to the future

\section{The silver lining of COVID-19 - Improving operational processes}

\author{
Sabina Cerimagic and Dewa Wardak \\ University of Sydney
}

\begin{abstract}
The COVID-19 pandemic has highlighted the need for improvement in our operational processes. In our Business Co-Design (BCD) team we have successfully utilised a design-based research (DBR) approach for the past two years - and it has worked well. However, we have noticed that from an operational perspective, the DBR approach methodology we have been using can struggle to scale up, as designing is bespoke and offers tailor made solutions for our individual projects. We will pilot incorporating elements of a soft systems thinking approach together with actornetwork theory (ANT) to enable us in applying a more holistic approach to the design and development of units of study which will provide opportunities for input from the larger team, instead of working in silos. Additionally, we are hopeful that this new approach will also assist us in making our design and projects scalable and sustainable - and to support us in increasing efficiency.
\end{abstract}

Keywords: teamwork, design-based research, system thinking, actor-network theory, improving processes and communications

\section{Introduction}

Prior to COVID-19, much of teaching and learning in higher education was delivered face to face or in blended modes with a relatively smaller number of courses offered fully online. The key dilemma posed by the COVID crisis was: How to rise to the challenge of improving the teaching and learning experience for both students and academics at short notice and at scale? At the University of Sydney Business School, a strategic project to transform the learner experience had just begun, the Connected Learning at Scale (CLaS) project. The Business Co-design (BCD) team was formed as part of this project. The BCD team is multi-disciplinary and consists of educational developers (ED), learning designers (LD), media producers, learning technologists, research evaluation associates (R\&E), and teaching online support staff (Cerimagic et al., 2020). The BCD teamwork with the Unit Coordinators (UC) and the teaching team to redesign existing units or design brand new units. $\mathrm{BCD}$ also provide support and advice on innovative course design, evaluating teaching and the impact on student learning, leadership in learning and teaching. We also provide support for experiential modes of teaching such as hybrid and gamified, just to name a few examples. Additionally, we pilot emerging and innovative pedagogical approaches.

At the start of the CLaS project, the BCD team worked on nine large core units of study at the Business School (with a maximum class size of 3,000) and begun to transform them through embedding three core CLaS principles, all informed by the importance of developing and leveraging community and connections. The project is built on redesigning the student experience in these large classes in line with the three CLaS principles (Vallis, et al. 2019).

- Principle 1: Information engagement - where students both individually and collectively engage with discipline knowledge as opposed to having it broadcast at them in a lecture.

- Principle 2: Connected participation and active learning -where face-to-face teaching time, student learning activities and technology are leveraged to build connections and networks to address, debate and solve critical global and local challenges though innovative pedagogical approaches. 
- Principle 3: Relevant and authentic assessment and feed-forward - where learning is applied and tested through authentic assessment modes supported by opportunities to receive and share feedback from both academics and their peers.

In this paper we will describe the changes that we are currently piloting, in light of contemporary conceptual frameworks of Systems thinking and ANT.

\section{The current operational approach while working on some of our large units}

The Business Co-design (BCD) team works on several projects - our largest projects are the CLaS projects and in this paper, we will focus on our operational approaches to what we call the 'Deep' touch and 'Light' touch CLaS. Generally, BCD educational developers work collaboratively on a 'Deep' touch CLaS unit with the UC, an LD, and a media expert for two to three semesters. We also evaluate our interventions in the unit through the semester, thus we involve a research evaluation associate $(\mathrm{R} \& \mathrm{E})$ too.

Our activities range from one-on-one consultations, and development project support to professional development programs. Our Deep touch CLaS units are bespoke designs- which can be very labour intensive and not always the most effective and efficient way to work.

As mentioned earlier, we also offer support for 'Light' touch CLaS units. This approach is more sustainable, but it does not offer the same 'high quality' of outcomes for the units, as we only spend two months on the light touch CLaS units. Unlike in the Deep touch CLaS approach where every UC works with a subset of the BCD team that consists of an ED, LD, media support and a R\&E - for the light touch units our EDs work with approximately ten units each and all the Light touch CLaS units are supported by only one LD. Figure 1 outlines the roles everyone plays in the development of the Light touch CLaS units.

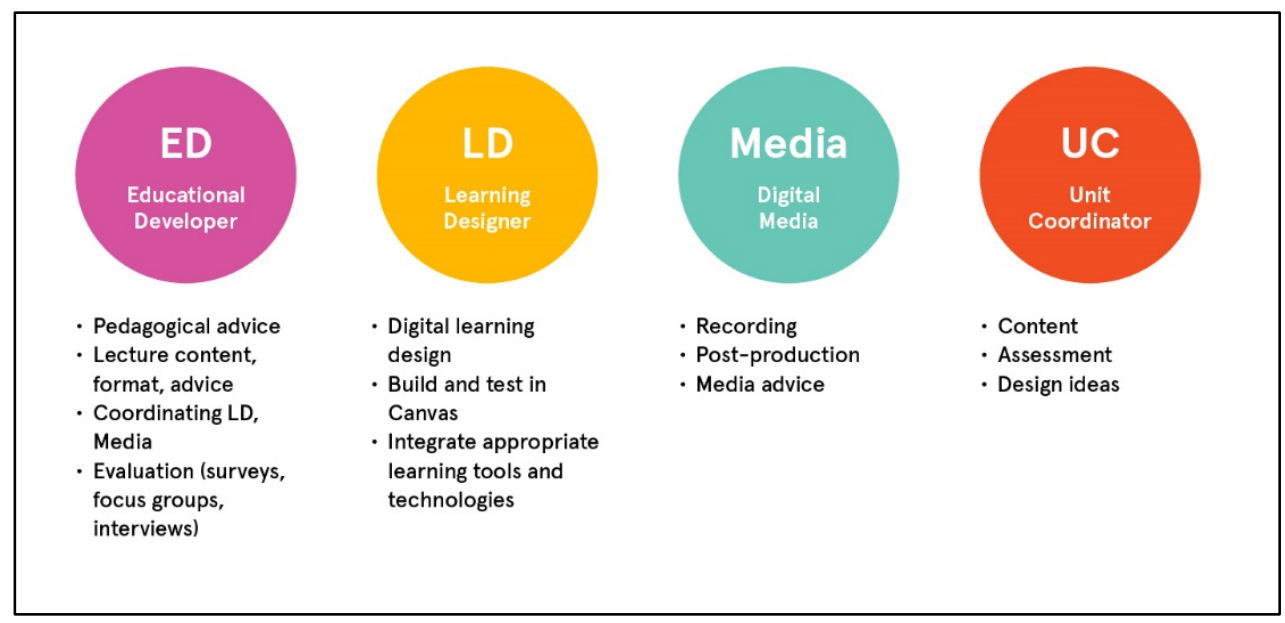

Figure 1. BCD - Light touch CLaS support model for scaling up

\section{Project scope - changes}

In the switch to remote learning, many higher education providers are scaling up their learning and curriculum design teams for online delivery (Vallis, et al 2019). The CLaS project started a year before the pandemic; however, due to the pandemic the BCD team's workload increased considerably from 2019 to 2021.

- 2019 - we worked with 9 Deep touch CLaS units

- 2020 - we worked with 16 Deep touch CLaS units and 35 Light touch CLaS units

- 2021 - we are working on 24 Deep touch and 15 Light touch CLaS units, with the possibility of more units being added throughout this year

Student feedback to the redesign of some units has been very positive. This led to the increase in demands from other UCs in the Business School who request the redesign of their units with the support of the BCD team. As a result, our work has increased exponentially without a commensurate increase in resources. Thus, we need to 
evaluate and improve our current operational approaches and processes to deal with this workload increase.

\section{Conceptual Underpinnings - the pilot support model}

We have successfully utilised a design-based research (DBR) approach for the past two years, and the general perception of students and faculty is that it has worked well. However, we have noticed that from an operational perspective, the design-based research approach methodology can struggle to scale up as our design is bespoke and offers tailor made solutions (Figure 2) for our Deep touch CLaS units (Dhukaram, et al., 2018; Seitzinger et al., 2020). This can create issues, such as each project that we work on could become a bespoke endeavour and it creates silos within the team; as well as, it can have negative effects on efficiency and sustainability.

Our aim is to continue to utilise the design-based research methodology given its well established benefits (Freeman, et al., 2014), but we will be building up on it by introducing and leveraging an overarching soft system thinking approach; as well as, the utilisation of Actor-Network Theory (ANT) for analytical purposes (Callon, 1986a; Hetherington, et al., 2000; Abend, 2008; Dhukaram, et al., 2018).



Figure 2. Current Deep touch bespoke design support model for individual units

Perceiving our 'units of study' as a system in themselves comprising of subsystems and nested within super systems, we hope that the introduction of soft system thinking, and ANT will enable us in applying a more holistic approach to the design and development of units of study which will provide opportunities for input from the larger team, instead of working in silos on individual units (Abend, 2008; Dhukaram, et al., 2018). Additionally, we also hope that this new approach will assist us in making our design and projects scalable and sustainable - and to support us in increasing efficiency.

\section{Defining soft systems thinking and ANT}

Systems thinking is a big-picture and comprehensive visualization method - in short, systems thinking is the approach beyond design thinking (Hetherington, et al., 2000; Mehregan, et al 2012; Pagano, et al, 2013).

Some scholars view systems thinking as a specific methodology, such as system dynamics, while others believe it is a plurality of methods. The literature on systems thinking indicates that system thinking is applied interchangeably with both systems science and systems theory. Systems thinking is also envisioned as a scientific orientation (a particular thinking style) and as a social movement (Freeman, et al., 2014; Dhukaram, et al., 2018). A minimalist definition of systems thinking is the study of systems of all types (Freeman, et al., 2014). In this pilot study we will be exploring and implementing the soft systems methodology for "increased capacity for communication, greater complexity of goals, and weaker correlation between power and authority" (Pagano et al., 2013). Systems thinking consists of mapping the system where our challenges live, 
understanding the different stakeholders (both internal and external) and their different agendas, mental models and relationships.

In our research, soft system thinking and DBR are the overarching frameworks while ANT will be used as an analytical tool to bring to light some of the taken-for-granted practices that need to be changed and updated (Abend, 2008; Freeman, et al., 2014). ANT can be both a theoretical and a methodological approach. It is about understanding human interactions with non-human entities. It clarifies complexities in a system, where the system can be conceptualised as an organisation. According to ANT, everything exists in networks of constantly shifting relations. It is often used to analyse the role of technology and how it shapes social relations (Callon, 1986a; Hetherington, et al., 2000; Abend, 2008). ANT assumes that social relations do not exist in isolation and that these are part of heterogenous networks of human and non-human entities (Abend, 2008).

We will be piloting the new approaches on seven new Capstone units that we will be working on as a team from mid-June 2021 to mid-June 2022.

\section{The Pilot}

We will be piloting the incorporation of elements from the soft systems approach, using ANT as an analytical method. To streamline our operational processes and to evaluate if this new approach works/is effective and worthwhile utilising on the seven brand new Capstone units, over the next 12 months.

The Capstone units will implement intentional design and development using a form of soft systems thinking and DBR approaches, and we will utilise ANT for its analytical capabilities. The realisation that we need to streamline processes led us to think that perhaps we need a more holistic team-based approach to the design and development of units of study that goes beyond the linking, planning, and patterning of various components to meaningfully understanding boundaries, perspectives and relationships and impacts of curricular elements.



Figure 3. Proposed/planned support model

Adapting Deming's (2014) work on quality management and Seddon and Caulkin (2007) combine aspects of lean (Lean Enterprise Research Centre), with more specific aspects of soft systems thinking (Zokaei et al., 2010), we are proposing a "Check, Plan, Do" cycle for systems thinking (Figure 3). The first stage ("Check") involves understanding the organisation as a system, the second stage ("Plan") identifies levers for effective change, and the third stage ("Do") implements direct action on the system. Since it is a cycle, the process is iterative with incremental changes applied in phases (Zokaei et al., 2010). 


\section{Steps in Reframing Capstone Units}

Step 1:

- We are starting with a whole-team approach including the EDs, LDs, the media team, and the R\&Es to brainstorm ideas. In monthly meetings, we will discuss a common ED and LD approach and incorporate feedback from Media and R\&E.

Step 2:

- The EDs will meet with the UCs to scope out the project, including any specific request and ideas the UC may have. This stage also involves an evaluation of the UC level of experience in using our Learning management System (LMS) and other technological tools.

- After the initial meeting, each ED/LD team will set up regular catch-up meetings with the UC.

Step 3:

- The whole-team meetings approach will continue, where the BCD team will have an opportunity to share progress and lessons learned from the design and development process. These meetings will also include external collaborators, such as work placement management staff at the faculty, who will work with the development teams for each unit on ideas for work-integrated learning opportunities for the Capstone units. This approach will enable each allocated ED to update their UC on the broader design approach, inform them of new tools and strategies, and opportunities for sharing resources between the Capstone units.

Step 4:

- We will have a showcase twice a year where we invite the academics we have worked with and showcase the Units to the entire faculty and the management team.

To support communication and sharing of resources and ideas between the Capstone design and development teams, we have created a MS-Teams site with channels dedicated to each unit. Each of the Capstone units also has a dedicated private channel to work with their own sub-team of Educational Developers (ED), Learning Designers (LD), research evaluation associates (R\&E) and unit coordinator (UC). This approach will facilitate the sharing of information, issues, challenges, and solutions with the wider team, to promote transparency, and encourage collaboration amongst the BCD team and the academics we are working with.

\section{Conclusion}

In this paper, we shared our plan for expanding our current design-based research approach to include elements of soft systems thinking and actor-network theory which will enable us to apply a holistic broad team-based approach to the design and development of large units of study at the Sydney Business School. As our work increased exponentially without a commensurate increase in resources, we are finding it inefficient to offer bespoke and tailor-made solutions for each of our individual large units.

This new more comprehensive approach will assist us in making our design and projects scalable and sustainable, and to support us in increasing efficiency. In this paper we shared our plan for employing this multidimensional approach in the design and development of seven new Capstone units where we will incorporate feedback and involvement from the larger BCD team in several stages throughout the year-long Capstone project. Future publications from this project will provide updates about the progress, achievements and lessons learned from this approach for Business programs aspiring for similar changes. The changes that we hope to achieve are worth doing and continuing with, post COVID-19.

\section{References}

Abend, G. (2008). The meaning of 'Theory'. Sociological Theory 26(2): 173-199.

Callon, M. (1986a). The sociology of an actor-network. In Mapping the dynamics of science and technology, Edited by: Callon, M., Law, J. and Rip, A. 19-34. London: Macmillan.

Cerimagic, S., \& Khanna, P. (2020). Higher Education Curriculum Renewal Through Systems Thinking. Pecha Kucha Presentation. Australasian Society for Computers in Learning in Tertiary Education (ASCILITE), 2020 conference. 30th of November and 1st of December 2020, University of New England, Armidale, NSW, Australia. 
Dhukaram, A. V., Sgouropoulou, C., Feldman, G., \& Amini, A. (2018). Higher education provision using systems thinking approach - case studies. European Journal of Engineering Education, 43(1), 3-25.

Freeman, R., Yearworth, M., \& Cherreault, J.Y. (2014). Review of Literature on Systems Thinking and System Dynamics for Policy Making. Technical Report, January 2014.

Hetherington, K. \& Law, J. 2000. After networks. Environment and Planning D. Society and Space, 18. 127130.

Mehregan, M.R., Hosseinzadeh, M., \& Kazemi, A. (2012). An application of soft system methodology Procedia. Social and Behavioral Sciences, 41(2012), pp. 426-433.

Midgley, G. (2000). Systemic intervention: Philosophy, methodology, and practice. Kluwer Academic Publishers, New York.

Pagano, R., \& Paucar-Caceres, A. (2013). Using systems thinking to evaluate formative feedback in UK higher education: the case of classroom response technology. Innovations in Education and Teaching International, 50(1), 94-103.

Seddon, J., \& Caulkin, S. (2007). Systems thinking, lean production and action learning. Action Learning Research and Practice, 4(1), 9-24.

Seitzinger J. (2020). Raising ABLE: Scaling Learning Design with a Design System. TELedvisors webinar. https://www.youtube.com/watch?v=3oOpawRk1j0

Vallis, C., Bryant, P., \& Huber, E., (2019). A CLaS on its own: Connected Learning at Scale, 36th International Conference on Innovation, Practice and Research in the Use of Educational Technologies in Tertiary Education (ASCILITE 2019).

Zokaei, K., Elia, S., O’Donovan, B., Samuel, D., Evans, B., \& Goodfellow, J. (2010). Lean and Systems Thinking in the Public Sector in Wales. Lean Enterprise Research Centre.

Cerimagic, S., \& Wardak, D. (2021). The silver lining of COVID-19 - Improving operational processes. In Gregory, S., Warburton, S., \& Schier, M. (Eds.), Back to the Future - ASCILITE '21. Proceedings ASCILITE 2021 in Armidale (pp. 230-235). https://doi.org/10.14742/ascilite2021.0134

Note: All published papers are refereed, having undergone a double-blind peer-review process.

The author(s) assign a Creative Commons by attribution licence enabling others to distribute, remix, tweak, and build upon their work, even commercially, as long as credit is given to the author(s) for the original creation.

(C) Cerimagic, S., \& Wardak, D. 2021 